Pramānạa - J. Phys., Vol. 35, No. 5, November 1990, pp. L503-L507. (C) Printed in India.

\title{
Possible observation of coulomb blockade at room temperature
}

\author{
G V SHIVASHANKAR and A K RAYCHAUDHURI \\ Department of Physics, Indian Institute of Science, Bangalore 560012, India
}

M S received 11 June 1990

\begin{abstract}
We have studied the (I-V) characteristics of the tunnel junction formed between the tip and the substrate in an STM at room temperature. We find that in such an arrangement it may be possible to get a junction capacitance $\simeq 10^{-19} \mathrm{~F}$ and junction conductance $<1 \mu \mathrm{s}$. When the junction conductance is $<1 \mu$ s strong nonlinearity is observed in the (I-V) characteristics. We explain this nonlinearity as onset of coulomb blockade of tunneling electrons.
\end{abstract}

Keywords. Tunneling; ultrasmall capacitance; tunnel junction; scanning tunneling microscope; coulomb blockade.

PACS No. $73 \cdot 40$

Discrete transfer of single electron in a tunnel junction of small capacitance $\left(C<10^{-15} \mathrm{~F}\right)$ is a topic of current interest [Likharev 1988; White et al 1986; Fulton and Dolan 1987]. Single electron charging effect has also been observed in an array of small metal particles [Cavicchi and Silsbee 1984; Mullen et al 1988; van Bentum et al 1988]. If the charging energy $e^{2} / 2 C \gg k_{B} T$ one observes effects arising out of discrete electron transfer. Generally it shows up in the (I-V) characteristics of the tunnel junction where one finds for $V \gg e / 2 C$,

$$
I=G(V-e / 2 C) \text {. }
$$

But for $V \ll e / 2 C$ one finds the conductance to be much less than $G$ and the current follows the relation,

$$
I=(2 C G / \pi e) V^{2} .
$$

The low conductance region arises due to blocking of further charge transfer when one electron has been transferred through the junction. This is generally known as coulomb blockade of tunneling electrons (Averin and Likharev 1986).

A very simple way to get an ultra small capacitance tunnel junction is to use a scanning tunneling microscope (STM) where the tip and the substrate forms a tunnel junction. It has been shown recently that in such a junction one can achieve a $C \simeq 10^{-18} \mathrm{~F}$ and $G \simeq 0 \cdot 1-1 \mu \mathrm{s}$ such that $e^{2} / 2 C k_{B}=10^{3} \mathrm{~K}$ and one is able to observe coulomb blockade at $T=4 \mathrm{~K}$ (van Bentum et al 1988).

A recent calculation (GeigenMuller and Schon 1988) shows that a clear observation of coulomb blockade at a finite $T$ is possible for $e^{2} / 2 C k_{B} T \geqslant 10$. If the ratio is lower than this the nonlinearity below $V=e / 2 C$ gets smeared out by thermal fluctuations.

In this paper we show that it is possible to achieve a tunnel junction with $C \simeq 10^{-19} \mathrm{~F}$ in an STM, so that $e^{2} / 2 C k_{B} \simeq 10^{4} \mathrm{~K}$ and it is possible to observe coulomb blockade even at room temperature. 


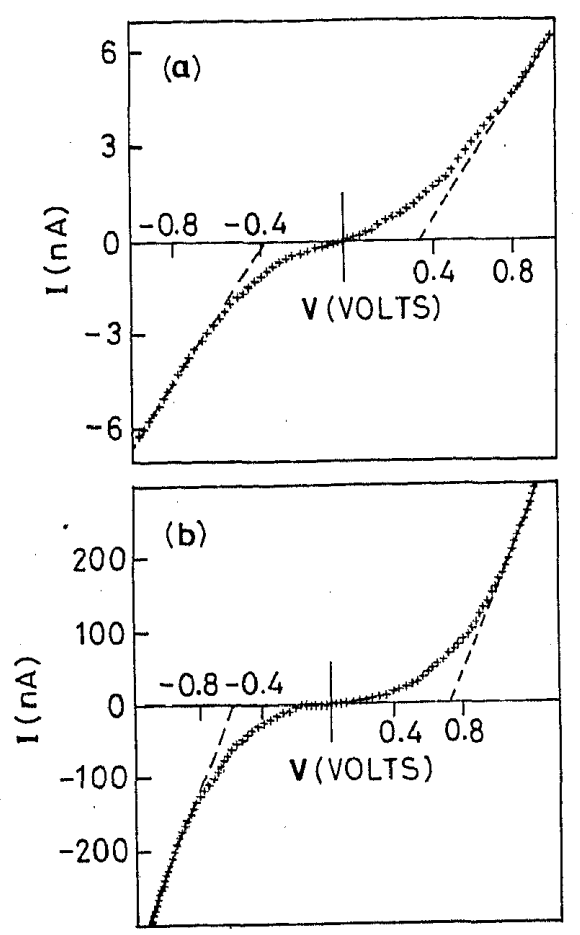

Figure 1. Typical observed (I-V) characteristics for the tunnel junction. For figure 1(a), $C=2.7 \times 10^{-19} \mathrm{~F}, G=10^{-8} \mathrm{~S}$ and for figure $1(\mathrm{~b}): C=1.3 \times 10^{-19} \mathrm{~F}, G=4 \times 10^{-7} \mathrm{~S}$. The dotted line in both figures show the linear relation (eq. (1)).

The experiment was carried out in a simple home made STM with standard piezodrives. The STM was operated at room temperature and in air. The tip was made from tungsten by electro-chemical etching and the substrate was a piece of stainless steel.

We recorded a large number of $(\mathrm{I}-\mathrm{V})$ curves by varying the substrate tip separation $(\Delta d)$. [The I-V scans were taken using Keithley Picoammeter (model 485) and Keithley DMM (model 197) and an IBM-PC compatible. Typical (I-V) scan lasts for 60 to 90 s depending on the voltage range and number of data points taken]. Two typical $(\mathrm{I}-\mathrm{V})$ curves are shown in figure 1 . One can see that though the tunnel current $\mathrm{I}$ in both the junctions are quite different the nonlinearity of the $(\mathrm{I}-\mathrm{V})$ at the low voltage region is quite pronounced in both these curves. [For $V<1.5 \mathrm{~V}$, the (I-V) curve is asymmetric within the noise of the data]. We find this nonlinear behaviour in all the (I-V) curves and the nonlinearity persists till the junction conductance reaches $\simeq 1 \mathrm{mS}$.

In order to see if the nonlinearity in the (I-V) curve arises due to coulomb blockade we fitted our data to eqs (1) and (2). The agreement of the data to the fit varies from good to reasonable. A typical example of the fit is shown in figure 2, where the data are shown in a log scale to enhance the lower I and V part. It is important to realize that the calculated curve has no fit parameter. Tunnel conductance $G$ and capacitance $C$ have been obtained from the asymptotic linear region using (1) and this $G$ and $C$ have been used to calculate tunnel current $I$ in the coulomb blockade region using (2). The agreement suggests that the observed nonlinearity may indeed be explained as coulomb blockade of tunneling electrons. 

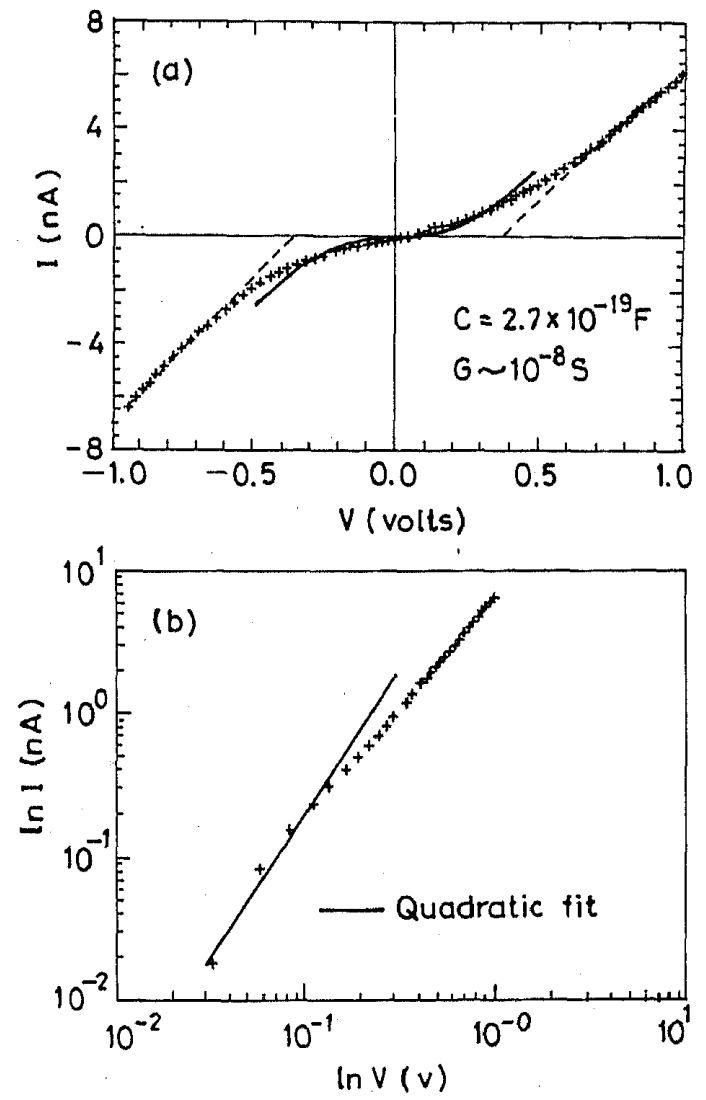

Figure 2. Calculated current, using the values of $C$ and $G$ (obtained from the linear asymptotes of the experimental curve of figure $1(a)$ ) in eq. (2) to obtain the current values in the coulomb blockade region. (a). Plot of calculated (I-V) fitted to the observed (I-V) curve in the low voltage region. (linear scale). (b). In $(I)-\ln (V)$ plot of figure 2(a). Solid line is the fit to the low voltage part.

The electro-chemical etching of the tip gives a typical tip head diameter of $<10 \mathrm{~nm}$ as confirmed by the atomic resolution of metal surfaces obtained using etched tungsten tips in a STM. Therefore a careful manipulation of the etched tungsten tip on a metal surface resulted in an ultrasmall tunnel junction. We also varied the junction resistance in order to obtain lower junction capacitance so as to observe coulomb blockade effects of tunneling electrons at room temperature. We find by varying the tip and substrate distance $(\Delta d)$, that for most curves $C=(1-3) \times 10^{-19} \mathrm{~F}$ and $G=10 \mu \mathrm{s}-$ $10 \mathrm{~ns}$. Since $G$ depends exponentially on $\Delta d$, a large variation in $G$ and small variation in $C$ is expected.

We suspect that the oxide layer on the surface determines $C$. One does not really go from a tunneling to a metallic point contact regime till the oxide layer is punched through and $G>1 \mathrm{~ms}$ is achieved. We see this in our experiment from the (I-d) curve (figure 3). It can be seen in (figure 3) that when $G<1 \mu$ s, the tunneling current $I$ depends exponentially on the tip displacement $(\Delta d)$.

For $G<1 \mu$ s the tip is not touching the oxide layer whereas for $G>1 \mu$ s the tip moves through the oxide (or it may elastically deform it first and when the strain is sufficient may eventually pierce it). When $G \geqslant 1 \mathrm{~ms}$ the oxide layer may be pierced 


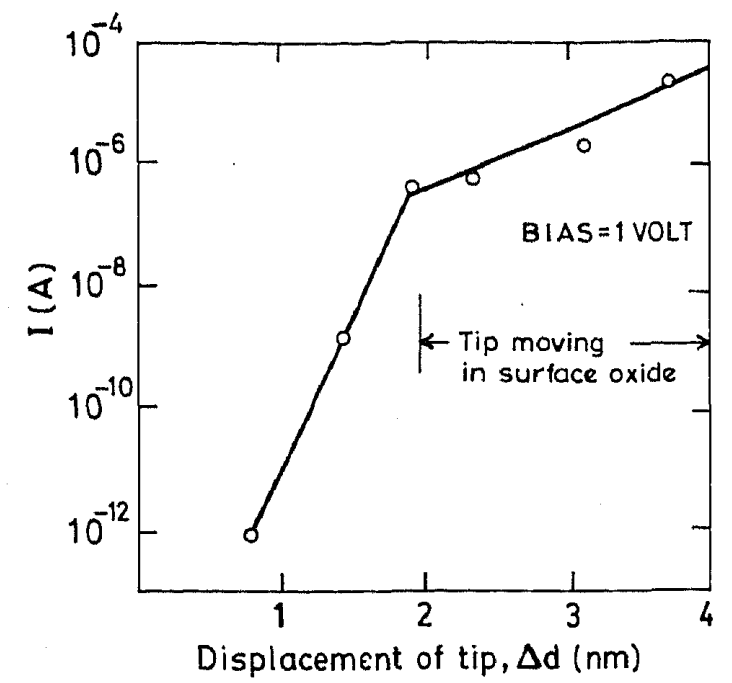

Figure 3. Dependence of tunnel current $I$ on the displacement $\Delta d$ of the tip. The displacement has been started from an arbitrary point where $I=10^{-12} \mathrm{~A}$. For $I=10^{-6} \mathrm{~A}$, the tip is believed to be moving through the oxide layer (see text).

through and one gets a metallic point contact. However another possibility may be that the tip is not moving straight up but forms an angle to the surface. At this point we can rule out the second possibility with certainty, since we observe that this bending over feature at high junction conductance is absent for platinum substrate where the slope of the (I-D) curve is quite stiff.

From the I-d curve in figure 3 we can get an estimate of the thickness of the oxide layer. As has been stated earlier, if we assume that when $1 \mathrm{~ms}>G>1 \mu$ s the tip is actually going through the oxide layer than a tip movement $d \simeq 3-4 \mathrm{~nm}$ is necessary to make $G$ change from $1 \mu \mathrm{s}$ to $1 \mathrm{~ms}$. The estimated thickness of the oxide layer is $\simeq 4 \mathrm{~nm}$.

The observed capacitance depends on the electric field distribution in the oxide and the space separating the tip and the oxide layer. The shape and dimension of the tip head, the point (or region) of the tip from where tunneling is taking place etc., will determine the capacitance. It is not known at present how one can estimate the capacitance taking care of all these factors. Since one can achieve atomic resolution using an STM tip of this type one thay expect that the effective tip head diameter $\simeq 10 \mathrm{~nm}$. (The effective tip head diameter is defined for the region over which electrons tunnel to or from the tip). For $d \simeq 1 \mathrm{~nm}$ this would imply $C \leqslant 10^{-19} \mathrm{~F}$. Thus a rough guess of junction capacitance of the order of $10^{-19} \mathrm{~F}$ is in reasonable agreement with the observed $C \simeq 2 \times 10^{-19} \mathrm{~F}$ obtained from the (I-V) curve. But we point out that the true capacitance $C$ is not simply given by $C=\varepsilon \varepsilon_{0} A / d$, where $A$ is the tip area and $d$ is the tip-surface distance.

To summarize, we have obtained in an STM a very low capacitance $\left(\simeq 10^{-19} \mathrm{~F}\right)$ tunnel junction and the nonlinearity in (I-V) curve suggests possible observation of coulomb blockade at room temperature. At this point we would like to point out that coulomb blockade is a suggested possibility. There are confirmatory experiments like observation of single electron tunneling (SET) oscillations (Guinea and Garcia 1990). It will be interesting to observe the SET oscillations at room temperature.

We thank K P Rajeev, Srikanth Hariharan and Pratip Mukhopadhyay for their kind cooperation regarding the experimental details and data acquisition and sharing their expertise. We also thank Prof. N Kumar for useful discussions. 


\section{References}

Averin D V and Likharev K K 1986 J. Low Temp. Phys. 62345

Cavicchi R E and Silsbee R H 1984 Phys. Rev. Lett. 521453

Fulton T A and Dolan G J 1987 Phys. Rev. Lett. 59109

GeigenMuller U and Schon G 1988 Physica B152 186

Guinea F and Garcia N 1990 Phys. Rev. Lett. 65281

Likharev K K 1988 I.B.M. J Res. Dev. 32144

Mullen K, Benjacob E, Jacklevic R C and Schuss Z 1988 Phys. Rev. B37 98

van Bentum P J M, Smokers R T M and van Kempen H 1988 Phys. Rev. Lett. 602543

van Bentum P J M, van Kempen K, van de Leemput L E C and Teunissan P A A 1988 Phys. Rev. Lett. 60369

White A E, Dynes R C and Garno J P 1986 Phys. Rev. Lett. 56532 